\title{
Tropospheric sounding with low-cost particulate matter sensors
}

\author{
Mark J. Potosnak*, Aarti Mistry ${ }^{\dagger}$ and Bernhard Beck-Winchatz \\ DePaul University, Chicago, IL, 60614 \\ Paul Ritter ${ }^{\S}$ \\ Pontiac Township High School, Pontiac, IL, 61764
}

\begin{abstract}
The high-altitude balloon (HAB) platform has allowed scientists to measure vertical profiles in the atmosphere at a relatively low cost. The current project combines the $\mathrm{HAB}$ platform with low-cost air quality sensors that measure particulate matter (PM). PM is detrimental to human health and can exacerbate asthma. In the atmosphere, PM can affect cloud formation and also radiative transfer, which links emissions of PM to climate change. Therefore, understanding and controlling PM emissions is vital to air quality and climate change. In agricultural regions, several practices produce significant PM emissions. Tilling can release PM in the form of dust, especially under arid conditions. The burning of crop residue is also a common practice practice that releases $P M$ in the form of partially combusted organics (soot). The ultimate goal of this project is to use low-cost PM sensors and HAB to assess PM sources from agricultural regions using citizen scientists. The current presentation evaluates the performance of two different PM sensors over flights conducted during the summer of 2017.
\end{abstract}

\section{Introduction}

$\mathrm{P}$ ARTICUlATE matter (PM) is a pollutant in the atmosphere that has both exposure health impacts and effects on climate change. Because of its relatively short residence time in the atmosphere, reductions in so-called black carbon emissions (e.g., soot) can quickly mitigate climate change and also improve regional health outcomes [1]. Satellites, in particular MODIS, can estimate column totals of PM through the measurement of Aerosol Optical Depth (AOD). Employing assumptions, models and empirical correlations, satellite measurements can be used to infer surface PM [2]. But the vertical distribution of PM is key for understanding the relationship between AOD and surface PM [3], and employing well-calibrated, low-cost sensors could improve satellite estimates. In particular, rural measurements of PM profiles would be useful for characterizing the background PM, which is necessary for interpreting urban surface PM retrievals from satellites such as MODIS [4].

While background profile data will be useful, the current project is further motivated by searching for point sources of PM in the agricultural landscape. For example, biomass burning, which is still practiced in the Midwest, and tilling for agriculture are both significant sources of PM [5]. These PM sources differ in the nature of the PM emitted. Tilling produces larger particle sizes ( $\mathrm{PM}_{10}$, diameter less than $\left.10 \mu \mathrm{m}\right)$ and more reflective mineral particles while biomass burning contributes smaller sizes ( $\mathrm{PM}_{2.5}$, diameter less than $2.5 \mu \mathrm{m}$ ) and more radiatively absorptive (black carbon) particles. These lead to differences in the ability of these particles to affect climate change (warming vs. cooling) and cloud properties. Other potential agricultural PM sources that could be investigated are emissions from heavy equipment and trash combustion. All this motivates considering the performance of low-cost PM sensors for making profile measurements.

\section{A. Previous research}

Initial research in 2016 produced some promising results with low-cost sensors. Test flights were conducted with a low-cost system [6, see Fig. 1]. Costing less than \$250, the system consisted of an Arduino with a GPS/SD card shield and a Sharp dust sensor tracked with a SPOT device. The decrease in PM density with altitude matches the decrease in air density. The spikes in PM near the ground during the descent are probably due to condensation, and generally descent data are not as useful due to turbulence. But the spike during the ascent in the second flight is interesting and

\footnotetext{
*Associate Professor, Department Environmental Science and Studies, $1110 \mathrm{~W}$ Belden Ave

†Undergraduate student, Department of Environmental Science and Studies, 1110 W Belden Ave

†Professor, STEM studies and Physics, 990 W Fullerton Ave

${ }^{\S}$ High school teacher, 1100 Indiana Ave
} 
potentially shows the capability of the system to detect interesting excursions in PM values. Another possibility is that the device ascended through a cloud, and water droplets were detected.

Provided with suitable training (workshops, videos, documents, see below), citizen scientists can use a hunt approach to characterize visible plumes of PM pollution. As long as certain guidelines are met (for example, total package weight is less than $12 \mathrm{lbs}$ ), FAA rules given in Part 101 do not require notice or other requirements that would be difficult for amateurs to meet. Given the lightweight equipment and the small balloons necessary, citizen scientists could travel through agricultural regions and find PM sources. The hunts would be targeted in time. For example, tilling typically occurs during the spring. The citizen scientists would use a series of launches, starting from the source to a distance where the plume is no longer visible. Measuring the plumes would enhance our knowledge of how agricultural sources contribute to the spatial heterogeneity of PM, which is missed by satellite retrieval algorithms.

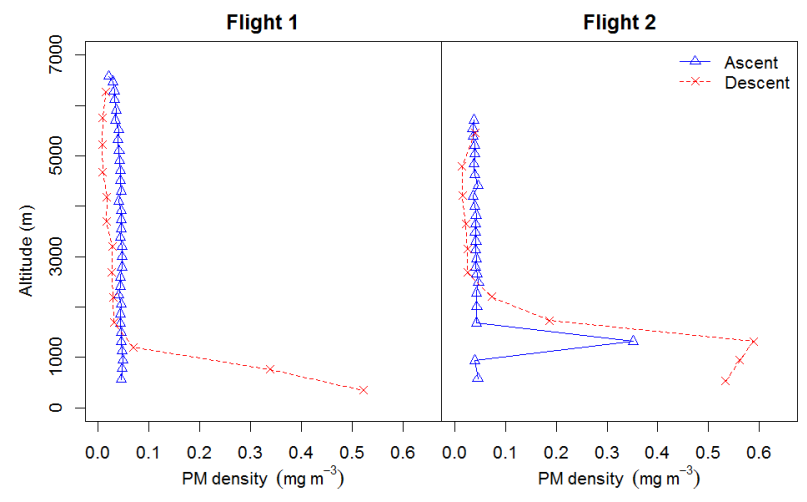

Fig. 1 Flights conducted during 2016 [6].

This science objective will be attractive to citizen scientists since it allows them to explore the impacts of food systems on regional air quality, and through the radiative properties of aerosols, climate change. In addition, the hunting of PM sources adds an element of excitement, which further engages citizen scientists. Because hunting for sources can lead to disappointment if none are found on a particular day, a backup plan would be to assess power plant plumes with carbon dioxide and PM sensors. By chance, one carbon dioxide flight in 2010 descended through the plume of the Schahfer Generating Station, one of the larger coal-fired power plants in the Midwest (Fig. 2). Citizen scientists could investigate both the carbon dioxide and PM emissions from sampling the plume. A relatively simple dilution model that accounted for the carbon dioxide source strength using published emissions data could be used to verify published PM emissions. Finally, citizen scientists could also explore long-range PM transport events. For example, distant wildfires in the western US and Canada can reach the Midwest with visible impacts in the atmosphere. But all these interesting projects require an assessment of the abilities of various low-cost PM sensors as deployed by the HAB platform.
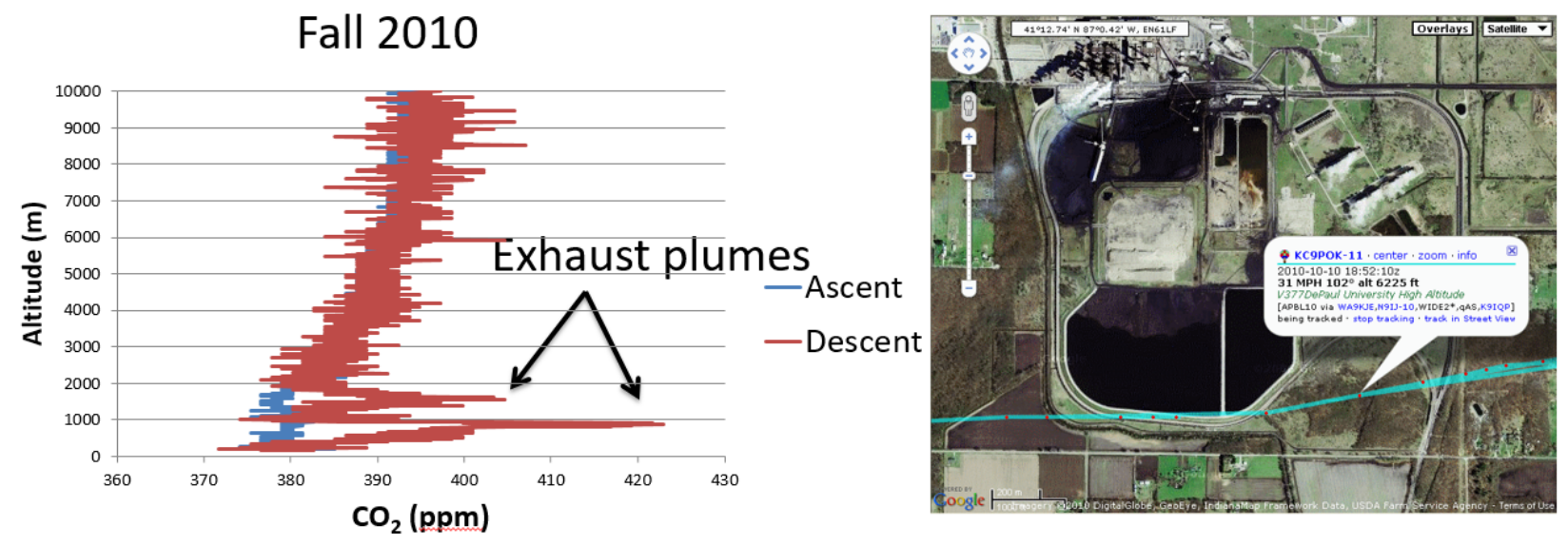

Fig. 2 Capturing a plume of $\mathrm{CO}_{2}$ from a power plant during a flight in 2010. 


\section{B. Research questions}

In the summer 2017, a more expensive (\$500), but still low-cost sensor was acquired. Two goals were established to further the project. First, the original low-cost sensor used during the summer of 2016 would be compared to the new, moderately more expensive sensor. Second, the particulate matter sensors would be compared to other chemical species. In particular, previously published research showed that carbon dioxide profiles can be measured with the HAB platform to evaluate the surface exchange of carbon dioxide [7]. By measuring carbon dioxide and PM profiles simultaneously, the quality and utility of the PM measurements can be assessed. While not as well studied and subject to more variability [8], ozone profiles were also evaluated. Unlike carbon dioxide, ozone can be produced in situ via chemical reactions at significant rates. Carbon dioxide is essentially stable once emitted. These two goals were pursued during one flight in June and with a series of four flights in September.

\section{Methods}

Most of the balloon launch and tracking procedures were similar to the methodology described previously [7, 8]. Briefly, a 150-gram latex balloon (Kaymont Industries, Melbourne, FL, USA) was filled with industrial-grade helium to achieve sufficient lift to produce ascent rates in the 4-5 $\mathrm{m} \mathrm{s}^{-1}$ range. A 6-foot parachute (Rocketman Parachutes, Bloomington, IN, USA) was used to control the descent. Tracking was accomplished with a Stratostar GPS command module (Noblesville, IN, USA). As a backup, flights were also tracked via the Automated Packet Reporting System (APRS) network using a GPS/radio combination (BigRedBee, Lake Oswego, OR, USA) and a lightweight hiking tracker (SPOT Gen3, Covington, LA, USA).

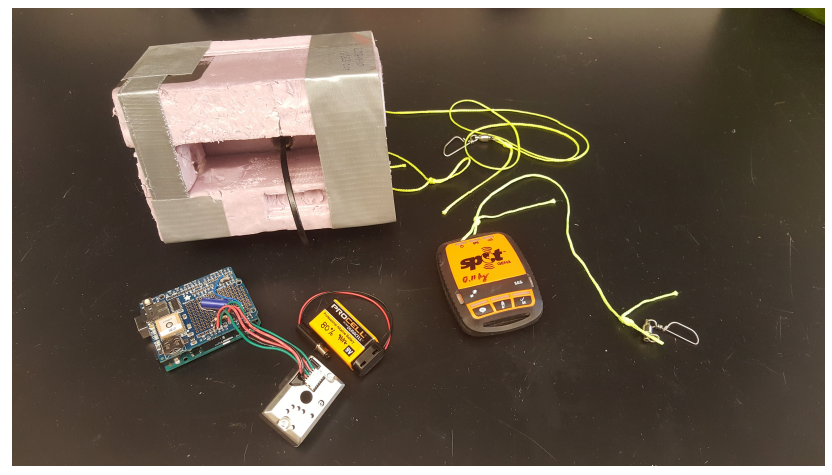

Fig. 3 Low-cost Sharp PM sensor used for the Jun flight.

All flights were conducted during the summer of 2017 (Table 1). On Jun 19, one flight was performed to compare the PM sensor used in 2016 with a new, more expensive system. The original sensor is very low cost: the sensor itself is only $\$ 11.95$ (Sharp, Optical Dust Sensor - GP2Y1010AU0F, Sparkfun Electronics, Niwot, CO, USA). The sensor was logged with an Arduino Uno board and a GPS shield (SparkFun GPS Logger Shield) which provided altitude information (Fig 3). The data rate was $1 \mathrm{~Hz}$. The new sensor uses a laser instead of infrared scattering to detect particles (OPC2, Alphasense, Great Notley, Essex, UK). In addition to better precision, the sensor separately reports different size classes of particles: $\mathrm{PM}_{10}, \mathrm{PM}_{2.5}$ and $\mathrm{PM}_{1}(10,2.5$ and $1 \mu \mathrm{m}$ diameter, respectively). The Alphasense has a rapid integration time, and a new measurement is recorded every $1.4 \mathrm{~s}$. The Jun launch occurred from the athletic fields of Pontiac Township High School $\left(40.886^{\circ} \mathrm{N}, 88.616^{\circ} \mathrm{W}\right)$. The flight traveled approximately $80 \mathrm{~km}$ to the east and ascended to $12.8 \mathrm{~km}$. The Sharp sensor failed at $7.8 \mathrm{~km}$, but since this project is focused on PM near the surface (up to $3 \mathrm{~km}$ ), this was not a serious issue.

The two PM sensors are both optical, but operate on different principles. The Sharp sensor uses an infrared LED source and a phototransitor detector. The detector is oriented at a right angle to the infrared LED, and the amount of scattered IR is assumed proportional to the PM content of the air. The manufacturer's calibration between voltage and dust density was used, as no calibration standard was available. The path of the detector has a diameter of $0.8 \mathrm{~cm}$ and a length of $1.76 \mathrm{~cm}$. Airflow through the detector path is passive. The Alphasense uses a laser to count particles in different size bins. The Alphasense software includes a density assumption that converts particle counts to particulate mass. There is an active fan that draws air into the Alphasense $\left(4-5 \mathrm{~mL} \mathrm{~min}^{-1}\right)$. Again, the factor calibration was used for the Alphasense. 


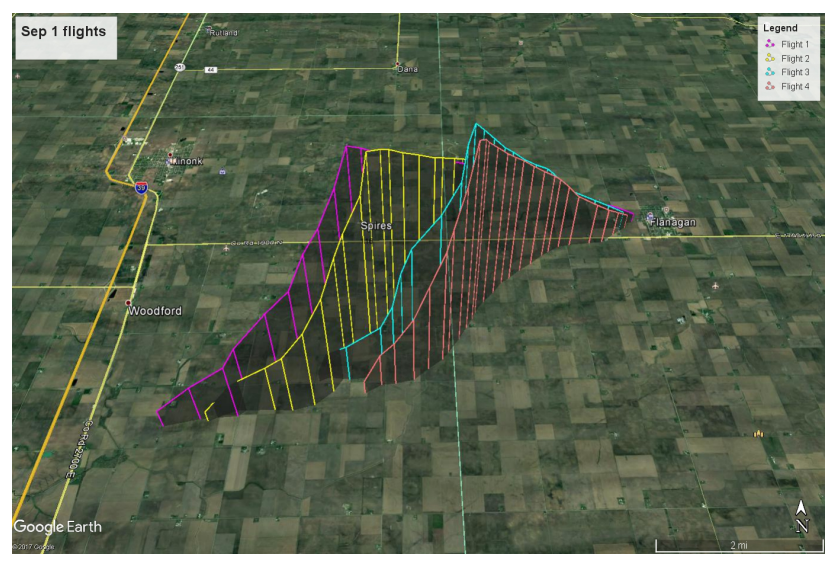

Fig. 4 Flights tracks for Sep 1, via the APRS system.

Table 1 Flight details

\begin{tabular}{lrrrl}
\hline Date & Flight & Time [CST] & Max alt [m] & Instruments \\
\hline Jun 19 & 1 & $14: 08$ & 12882 & Sharp PM, Alphasense PM \\
Sep 1 & 1 & $11: 08$ & 5798 & Alphasense PM, O, $\mathrm{CO}_{2}$ \\
Sep 1 & 2 & $12: 32$ & 5708 & Alphasense PM, O, $\mathrm{CO}_{2}$ \\
Sep 1 & 3 & $14: 33$ & 5766 & Alphasense PM, O O, $\mathrm{CO}_{2}$ \\
Sep 1 & 4 & $15: 52$ & 5683 & Alphasense PM, O, $\mathrm{CO}_{2}$ \\
\hline
\end{tabular}

On September 1, four sequential flights were performed with the Alphasense PM instrument, a carbon dioxide instrument [for details and picture of the flight packages, see 7] and an ozone sensor (Personal Ozone Monitor, 2B Technologies, Boulder, CO, USA). The POM had a modified pressure sensor for high-altitude work, and included an integrated pump, data acquisition system and GPS. The POM accuracy and precision are specified by the manufacturer as the greater of $1.5 \mathrm{ppb}$ or $2 \%$ of the reading, but this was not verified due to a lack of calibration source. The same balloon, parachute and tracking equipment were used as for the Jun flight. The flights were launched from a city park in Flanagan, IL $\left(40.877^{\circ} \mathrm{N}, 88.865^{\circ} \mathrm{W}\right)$. To reduce the flight time and allow for multiple flights, a cut-down device was deployed that activated at an altitude of approximately $6 \mathrm{~km}$. The device used an Arduino-type device and a pressure sensor (BMP280 I2C or SPI Barometric Pressure and Altitude Sensor, Adafruit, New York, NY, USA) to fire an e-match, which cut free the balloon from the parachute. Because of the cut-down device, flight distances were relatively short (about $10 \mathrm{~km}$, Fig.4).

The data analysis procedure was relatively simple. GPS altitudes were used for the Jun flight, and all data were averaged into 100-meter bins. For the Sep flight, GPS altitude and pressure were collected by the Stratostar command module, and the hydrostatic equation relationship was applied to derive altitude information from the pressure data collected with the carbon dioxide instrument [see details in 7]. Again, all data were averaged to 100-meter altitude bins.

\section{Results and Discussion}

The Jun flight was used to compare the two PM instruments. The results from that Jun flight demonstrated that while the Alphasense instrument has potential, the Sharp PM sensor is not adequate for tropospheric sounding work

\section{A. Jun flight: sensor comparison}

Results from the Jun flight are shown in Fig. 5. The top-left panel are data from the Sharp sensor, while the other three panels are PM observed with the Alphasense detector. Since those other three panels come from the same instrument, the tight correlation is not surprising. The Sharp data show very little structure. The results are similar to the previous summer's flight (Fig. 11). There is a noticeable decline with altitude. On the other hand, there is much more structure visible with the Alphasense instrument. One feature in particular stands out: PM concentrations for all three 


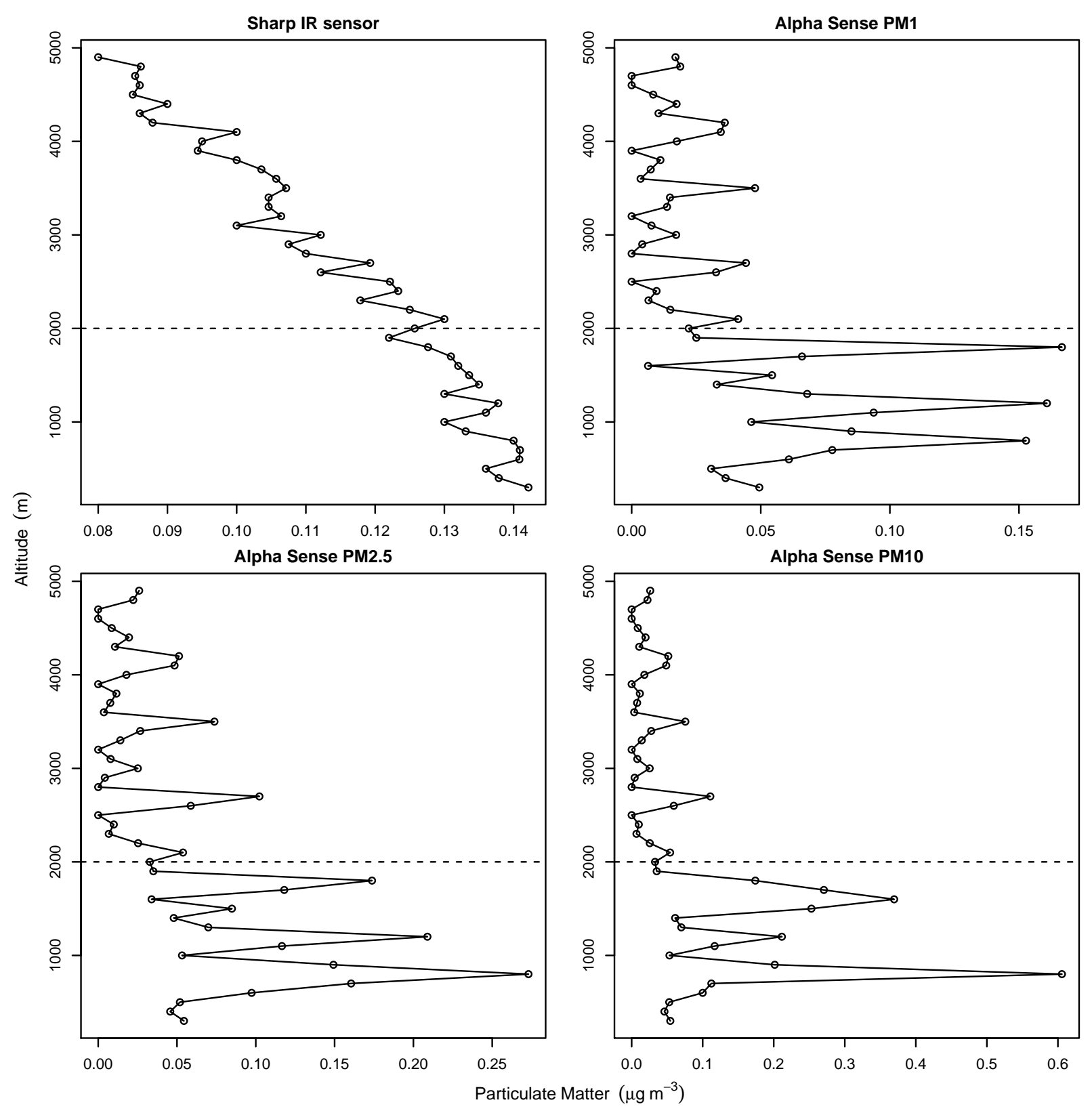

Fig. 5 Data from Jun flights which compared the two PM sensors.

size classes are higher below $2000 \mathrm{~m}$. To visualize this, a dashed line was drawn at this altitude. A feature like this makes sense from the point of view of boundary layer dynamics. Solar heating at the Earth's surface causes convection which forms a mixed layer of the atmosphere [9]. This mixed layer has relatively uniform properties that are distinct from the free troposphere above. For example, water vapor concentrations are typically much higher in the mixed layer.

Because PM sources have relatively short lifetimes in the atmosphere (days to weeks), concentrations are expected to be higher in the mixed layer. Further, the heavier particles ( $\mathrm{PM}_{10}$, bottom-right panel) should have an even shorter lifetime and larger concentration contrast across the top of the mixed layer. From these observations, it can be concluded that the Alphasense is producing a consistent dataset, but that the Sharp fails to capture this feature which should be observed. More work will be required to investigate if the distinct spikes in PM observed within and above the mixed layer are real features or are artifacts of the Alphasense instrument. 


\section{B. Sep flight: comparison to carbon dioxide and ozone}

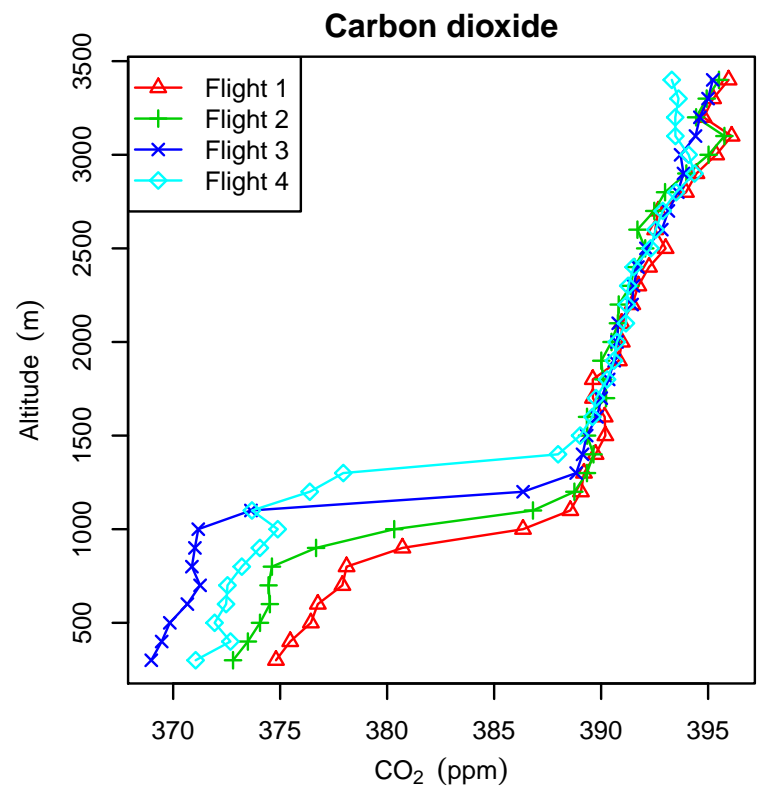

Fig. 6 Carbon dioxide profiles from the Sep flights.

The carbon dioxide data served its purpose. There is a readily identifiable top of the mixed layer observed during each flight (Fig. 6). Increasing from 800 to $1300 \mathrm{~m}$, the mixed layer is defined by the rapid increase in carbon dioxide. Below the mixed layer, carbon dioxide is being removed by plant photosynthesis. In comparison, the free troposphere above has higher carbon dioxide. The mixed layer increases in height during the day as solar heating continues to occur at the surface and causes increased mixing. The mixed layer heights determined from the carbon dioxide profiles can be used to interpret the data from the PM sensor.

Considering these carbon dioxide profiles in terms of surface exchange [7], the first three flights make sense. In each flight, the mixed layer height increases. The mixed layer has lower carbon dioxide compared to the free troposphere, since carbon dioxide is removed by plant photosynthesis. In addition, the mixed layer carbon dioxide concentration is decreasing from flights 1 to 3 . Again, this is consistent with plant uptake, as expected. For flight 4 , while the mixed layer height increases, the mixed layer concentration increases. One possible explanation is that plant photosynthesis decreased later in the day. Another possibility is that a local carbon dioxide source increased in strength. Looking at the flight tracks in Fig. 4, there is no evidence for a major shift in wind direction between the flights. When turning to the ozone and PM profiles, changes in flight 4 will be considered.

For ozone (Fig. 77), the profiles are more complicated and less consistent as compared to carbon dioxide. During flight 1 , there is a clear change in ozone across the top of the mixed layer $(1000 \mathrm{~m})$, but then there is little evidence for a sharp change during the other three flights. There are also changes between the flights in ozone concentrations above the mixed layer (for example, 3000 to $3500 \mathrm{~m}$ ). Note that there are also some small changes in carbon dioxide at this height, which might correspond to changes in the source of these upper-level air masses. But overall, while carbon dioxide is a stable gas in the Earth's atmosphere, ozone participates in atmospheric chemistry process. Ozone is created photochemically via reactions initiated by volatile organic compound and nitrogen oxide emissions. Ozone is also destroyed by reactions with other chemical species.

The results from these Sep test flights show that while ozone is very interesting, the profiles are much less constrained and will not be able to help elucidate the PM profiles. The one exception is during flight 4 . For carbon dioxide, flight 4 was noted as different since carbon dioxide increased instead of decreasing. The ozone concentrations for flight 4 are much lower than the other three flights. Again, since wind direction did not change, there was either rapid change in surface processes due to time of day or another local source capable of increasing carbon dioxide and decreasing ozone became stronger.

The Alphasense PM instrument collected all three size categories, but results were similar and only data from $\mathrm{PM}_{2.5}$ is shown (Fig. 8). The most notable feature is the sharp decrease in PM going from the mixed layer to the free 


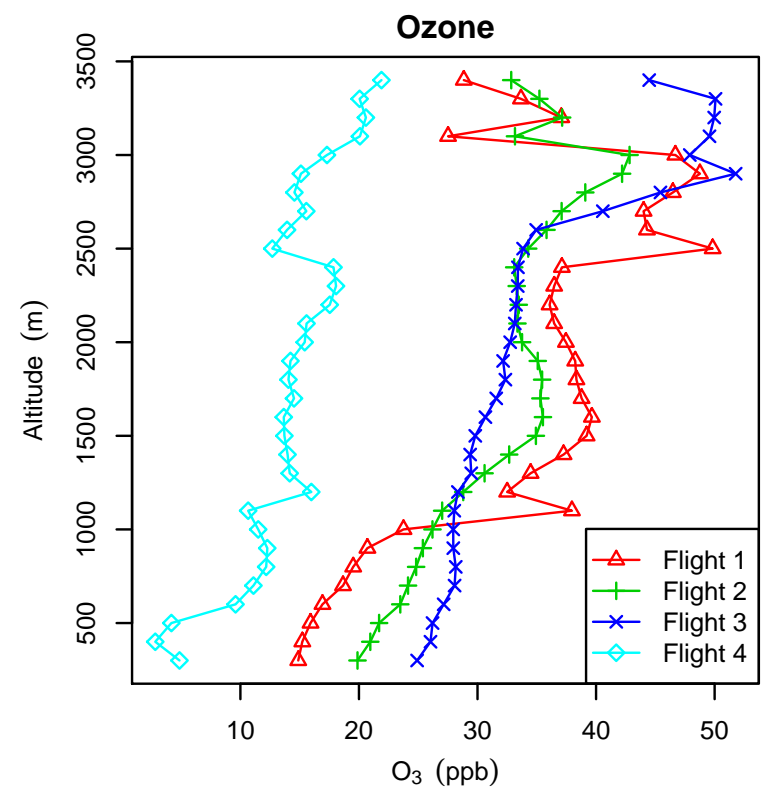

Fig. 7 Ozone profiles from the Sep flights.

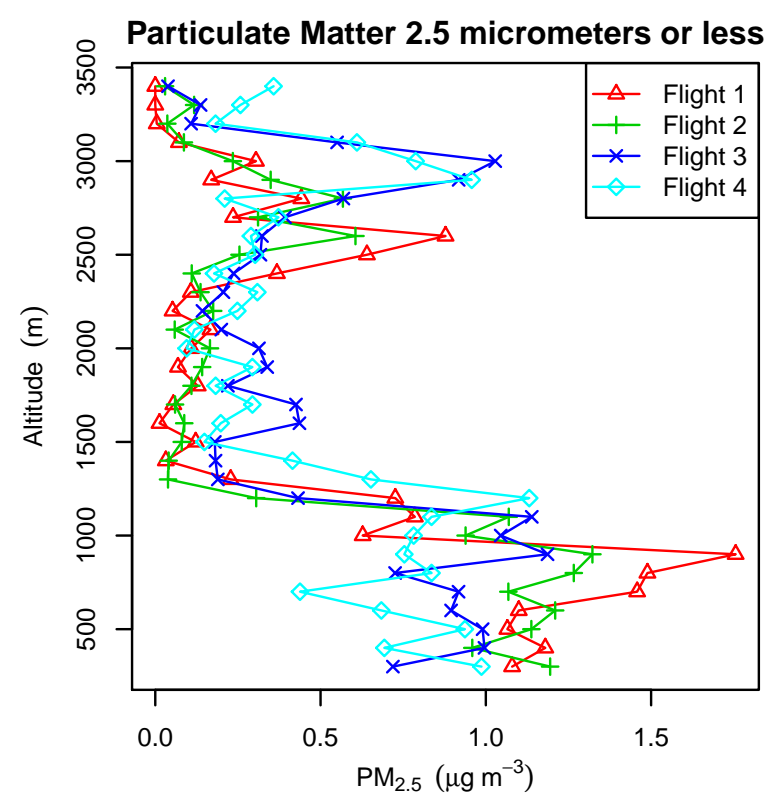

Fig. 8 PM profiles from the Sep flights.

troposphere. Again, as discussed for the Jun flight, this is expected because of the relatively short lifetime of PM in the atmosphere. Interestingly, unlike for carbon dioxide and ozone, there is nothing striking about flight 4 . While the concentrations are somewhat lower in the mixed layer, the effect is relatively small. Above the mixed layer, there is no discernible difference. Again, there are periodic spikes in the PM data at different altitudes. Of particular interest are the increases at 2500 to $3000 \mathrm{~m}$. These could potentially be layers of high PM from distant biomass burning or power plants.

Compared to the average concentration in the mixed layer, the sharp departures could be real features or could be due to instrument issues. For example, during flight 1 there is a significant increase around $800 \mathrm{~m}$, which from the carbon dioxide profile is the height of the mixed layer. Hypothetically, this could be high PM from nighttime sources. Again, 
more demonstrations will be necessary, but the Alphasense PM sensor has the potential for elucidating information about sources by measuring concentration profiles. In addition, more effort is necessary to check the calibration of the Alphasense as a function of air temperature and pressure and to evaluate if sun shielding or other airflow considerations are required.

\section{Conclusion}

Overall, the Alphasense instrument has good potential as a flight instrument. While more expensive than the Sharp PM instrument, the Alphasense is still relatively low-cost at $\$ 500$. The Sharp is a useful instrument for wearable air quality monitoring [10], but does not have the precision necessary for collecting profiles of PM in the mixed layer. The Alphasense has some advantages: the fan is built in and the device has its own data acquisition system. These are big advantages for citizen scientists. Also, the Alphasense can be powered by a standard micro-USB connector, so readily-available cell phone power packs can be used to provide power. One significant drawback is that the unit has no GPS and not even a real-time clock. So, an exact note of the power-up and power-down times must be recorded. Also, some other GPS/altimeter device will need to be flown for data analysis. But given these limitations, the Alphasense warrants further investigation for citizen science projects. While more costly, the use of a tethered-balloon system has great potential. As seen in Fig. 8, the top of the mixed layer was less than $1500 \mathrm{~m}$. From Bouche et al. [7], boundary layer heights were less than $2000 \mathrm{~m}$. A tether system could repeatedly measure PM profiles and provide information about how sources were varying in time. Another avenue of future research is comparing the advantages and disadvantages of balloon systems with unmanned aerial vehicle (UAV, "drone") platforms.

\section{Funding Sources}

The authors would like to thank the NASA-funded Illinois Space Grant Consortium for providing support for the earlier carbon dioxide work. The authors also received support from DePaul University's Academic Initiatives Program. The low-cost PM instruments were purchased for research on wearable air quality sensors with support from the Vincentian Endowment Fund at DePaul University.

\section{Acknowledgments}

The authors thank Pontiac Township Public High School for providing a launch location. They are also grateful for the amateur radio operators that make the APRS network possible. The authors thank Doreen Elrad for her help during the September 1 launch.

\section{References}

[1] Shindell, D., Kuylenstierna, J. C. I., Vignati, E., van Dingenen, R., Amann, M., Klimont, Z., Anenberg, S. C., Muller, N., Janssens-Maenhout, G., Raes, F., Schwartz, J., Faluvegi, G., Pozzoli, L., Kupiainen, K., Höglund-Isaksson, L., Emberson, L., Streets, D., Ramanathan, V., Hicks, K., Oanh, N. T. K., Milly, G., Williams, M., Demkine, V., and Fowler, D., "Simultaneously Mitigating Near-Term Climate Change and Improving Human Health and Food Security," Science, Vol. 335, No. 6065, 2012, pp. 183-189. doi:10.1126/science.1210026, URL http://science.sciencemag.org/content/335/6065/183

[2] Gupta, P., Christopher, S. A., Wang, J., Gehrig, R., Lee, Y., and Kumar, N., "Satellite remote sensing of particulate matter and air quality assessment over global cities," Atmospheric Environment, Vol. 40, No. 30, 2006, pp. 5880 - 5892. doi:https://doi.org/10. 1016/j.atmosenv.2006.03.016, URL http://wWW.sciencedirect.com/science/article/pii/S1352231006002858

[3] Li, J., Carlson, B. E., and Lacis, A. A., "How well do satellite AOD observations represent the spatial and temporal variability of PM2.5 concentration for the United States?" Atmospheric Environment, Vol. 102, No. Supplement C, 2015, pp. 260 - 273. doi:https://doi.org/10.1016/j.atmosenv.2014.12.010, URL http://www.sciencedirect.com/science/article/ pii/S1352231014009583

[4] Kloog, I., Koutrakis, P., Coull, B. A., Lee, H. J., and Schwartz, J., “Assessing temporally and spatially resolved PM2.5 exposures for epidemiological studies using satellite aerosol optical depth measurements," Atmospheric Environment, Vol. 45, No. 35, 2011, pp. 6267 - 6275. doi:https://doi.org/10.1016/j.atmosenv.2011.08.066, URL http://www. sciencedirect. com/science/article/pii/S1352231011009125.

[5] Hays, M. D., Fine, P. M., Geron, C. D., Kleeman, M. J., and Gullett, B. K., "Open burning of agricultural biomass: Physical and chemical properties of particle-phase emissions," Atmospheric Environment, Vol. 39, No. 36, 2005 , pp. 6747 - 
6764. doi:https://doi.org/10.1016/j.atmosenv.2005.07.072, URL http://www.sciencedirect.com/science/article/ pii/S1352231005007247

[6] Potosnak, M. J., Beck-Winchatz, B., and Ritter, P., "Low-cost HAB platform to measure particulate matter in the troposphere," Academic High-Altitude Conference, St. Paul, MN, 2016.

[7] Bouche, A., Beck-Winchatz, B., and Potosnak, M. J., "A high-altitude balloon platform for determining exchange of carbon dioxide over agricultural landscapes," Atmospheric Measurement Techniques, Vol. 9, No. 12, 2016 , pp. $5707-5719$. doi:10.5194/amt-9-5707-2016, URL https://www . atmos-meas-tech.net/9/5707/2016/

[8] Sabo, C., "Using a High-Altitude Balloon Platform to Observe and Measure Ozone Uptake over Agricultural Landscapes in Central Illinois," DePaul Discoveries, Vol. 5, No. 1, 2016. URLAvailableat:http://via.library .depaul . edu/depauldisc/vol5/iss1/18

[9] Stull, R., An Introduction to Boundary Layer Meteorology, Atmospheric Science Library, Springer, 1988.

[10] Abbate, J. M., “The Mobile Monitoring of Particulate Matter through Wearable Sensors and Their Influence on Students' Environmental Attitudes," DePaul Discoveries, Vol. 6, No. 1, 2017. URL Availableat:http://via.library.depaul. edu/depaul-disc/vol6/iss1/8 